\title{
Mehr Publikationen oder verbesserte klinische Forschung?
}

\author{
More Publications or Advanced Clinical Research?
}

U. Göbel

Bibliografie

DOI 10.1055/s-0028-1082059 Klin Pädiatr 2008; 220: 217-218

(c) Georg Thieme Verlag KG

Stuttgart . New York

ISSN 0300-8630

Korrespondenzadresse

Prof. em. Dr. U. Göbel

Heinrich-Heine-Universität

Düsseldorf

Moorenstr. 5

40225 Düsseldorf

Goebel@med.uni-duesseldorf. de
Die Universitätsklinika in Deutschland sind als Anstalten des öffentlichen Rechts verselbständigt oder durch Verkauf privatisiert worden. Als direkte Folge ist eine Trennungsrechnung zwischen den medizinischen Fakultäten und den Universitätsklinika begonnen worden, um die Zuweisungen der Länder für Forschung und Lehre unter Beachtung leistungsbezogener Aspekte gezielt und gerecht aufzuteilen.

Ein Beurteilungsparameter ist die publikatorische Leistung der Fakultätsmitglieder, mit der wichtige Beobachtungen oder Ergebnisse der klinischen bzw. experimentellen Forschung der interessierten Öffentlichkeit zugänglich gemacht werden. Hierzu wird für jede Fakultät der kumulierte Impact Factor (IF) zum Vergleich herangezogen, der einmal jährlich von Thomson Reuters (ehem. Thomson Scientific/ISI) in Philadelphia/ USA veröffentlicht wird. Mit diesem indirekten Parameter wird nicht eine Publikation selbst beurteilt, sondern die Zitierrate der Zeitschrift, in der eine oder mehrere Arbeiten des Autors publiziert worden sind. Um eine möglichst aktuelle Bewertung der publikatorischen Leistung zu erhalten, werden nur die während eines Kalenderjahres erfolgten Zitierungen von Arbeiten berücksichtigt, die innerhalb der vorangegangenen beiden Kalenderjahre erschienen sind.

Eine Kompetition besteht in doppelter Weise: Zum einen nimmt die Zahl der eingereichten Manuskripte zu und zum anderen wird zunehmend Wert auf die Höhe des IF der Zeitschrift gelegt. Beides führt dazu, dass die Einreichungen bei Zeitschriften mit steigendem IF überproportional zunehmen. Dies ist auch bei der Klinischen Pädiatrie zu beobachten, da seit 2004 der IF ansteigt ( $\bullet$ Abb. 1) und sich die Zahl der jährlich eingereichten Arbeiten zwischenzeitlich etwa verdoppelt hat. Dieser positive Aspekt hat zwangsläufig zu einer höheren Ablehnungsrate geführt, da die Seitenzahl der Zeitschrift nicht entsprechend erhöht werden konnte.

Der Anstieg des IF der Klinischen Pädiatrie für das Jahr 2005 wurde vorzugsweise durch vorausgegangene redaktionelle Veränderungen und mehr Publikationen in englischer Sprache erklärt [1]. Die nachfolgende Stagnation für das Jahr 2006 wurde auf die Veröffentlichung einer größeren Zahl von Übersichtsartikeln zurückgeführt, die häufig gelesen, aber nur selten zitiert werden [2].
Umso erfreulicher ist zu vermerken, dass sich der positive Trend der Vorjahre wieder eingestellt hat und der IF von 1055 im Jahr 2006 auf 1321 für das Jahr 2007 angestiegen ist.

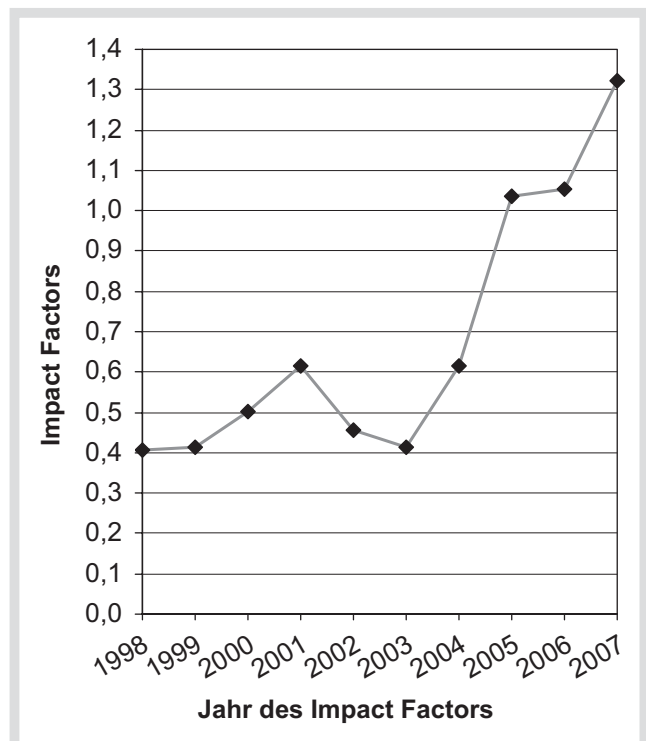

Abb. 1 Klinische Pädiatrie: Entwicklung des Impact Factors.

Allerdings handelt es sich hierbei nicht um ein isoliertes Phänomen für die Klinische Pädiatrie, wie das Ranking im Vergleich zu den anderen gelisteten Zeitschriften zeigt ( $\triangle$ Abb. 2). Obwohl der IF von 1034 für 2005 auf 1055 für 2006 noch leicht anstieg, fiel die Klinische Pädiatrie im Ranking von Platz 40 auf Position 44 zurück. Die jetzt mit dem IF von 1321 erreichte Position 37 ist nur wenig besser als die von 2005. Allerdings hat sich in der Zwischenzeit die Zahl der gelisteten Journale leicht von 73 auf 78 erhöht, sodass der Gesamteindruck für 2007 günstiger ist.

Diese Beobachtung zeigt, dass in den zurückliegenden Jahren weltweit die Kompetition zugenommen hat und für den gleichen Rang im internationalen Vergleich ein immer höherer IF benötigt wird. Hieraus nährt sich die Hoffnung, dass die Qualität der pädiatrischen Veröffentlichungen in den gelisteten Zeitschriften besser geworden ist und dies als indirekter Hinweis für eine verbesserte Forschungsaktivität gewertet werden könnte. Kritisch dagegen einzuwenden ist, dass 


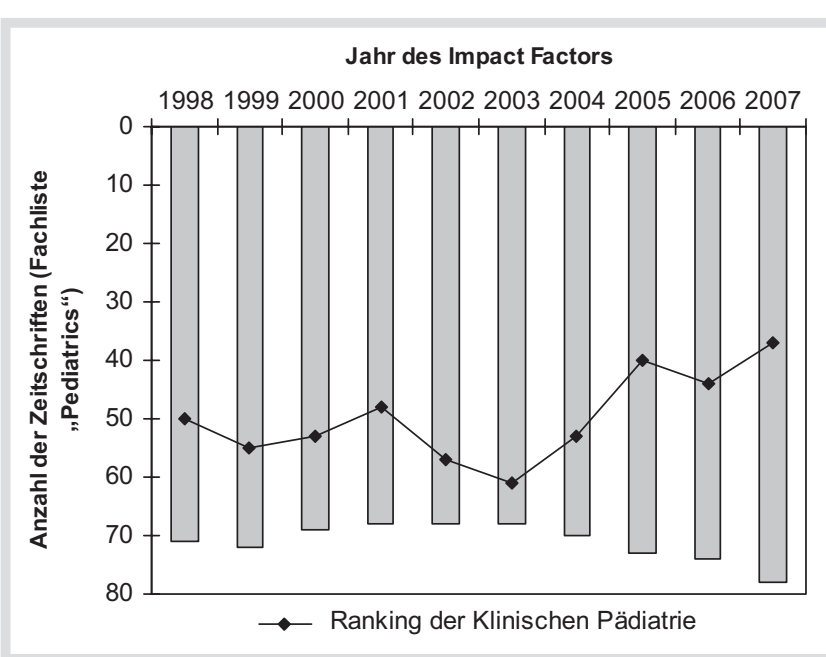

Abb. 2 Entwicklung des Rankings der Klinischen Pädiatrie innerhalb der Fachliste „Pediatrics“ der Thomson Reuters Journal Citation Reports ${ }^{\circledR}$.

es durch die vermehrte publikatorische Tätigkeit zu einer Selektion der Arbeiten in den gelisteten Journalen gekommen ist, die häufiger zitiert werden.

Für diese Annahme spricht, dass die von Thomson Reuters gelisteten Journale nur eine Minderzahl aller medizinischen Zeitschriften mit Wissen vermittelndem Anspruch darstellen. So lässt sich leicht für die Pädiatrie im deutschsprachigen Raum eine recht lange Liste von periodisch erscheinenden Zeitschriften mit ISSN-Nummer bilden, von denen jedoch nur zwei im internationalen Ranking erscheinen.

Unverrückbare Ziele einer engagierten Forschungs- und Publikationstätigkeit in der Medizin, so auch in der Pädiatrie, sind ein besseres Verständnis der Ätiologie und Pathogenese von Erkran- kungen sowie die Krankheitsprävention bzw. die verbesserte Diagnostik und Therapie manifester Erkrankungen. Dabei wird die Kuration einer Erkrankung in möglichst wenig belastender, aber immer erfolgreicherer Weise angestrebt, was mittels valider biometrischer Testverfahren vergleichend beurteilt werden kann. Um sich nicht mit punktuellen Vergleichen begnügen $\mathrm{zu}$ müssen, werden die Patienten in Register aufgenommen und langfristig nachbeobachtet.

Register sind bei seltenen Erkrankungen oder neuen Therapieverfahren besonders geeignet, um die Dauerhaftigkeit der Behandlungen nachzuverfolgen oder unerwünschte Spätfolgen möglichst rasch zu erkennen. Dies setzt interdisziplinäre Vernetzungen voraus, die der genauen Definition und Beschreibung bedürfen. Der Patient bemisst allerdings den Erfolg all dieser Aktivitäten und persönlichen Belastungen für sich an seiner Lebensqualität. Dies zeigt, dass sehr unterschiedliche Bewertungsskalen in der Medizin nebeneinander stehen, die alle eine rationale Basis haben und sachgemäßer sind als ein IF.

Die unverkennbaren Fortschritte der medizinischen Behandlungen wie auch die immer wieder zu verzeichnenden Rückschläge erfordern eine schriftliche Darstellung, die den immer stringenteren wissenschaftlichen Kriterien gerecht werden müssen. Die zunehmende Interdisziplinarität der klinischen Forschung mit ihren neuen Strukturen und die Kompetition bei der Ressourcenzuteilung führen nicht nur zu mehr, sondern auch zu besseren Publikationen.

\section{Literatur}

1 In eigener Sache - Impact Factor 2005: 1.036. Klin Pädiatr 218; 247-248

2 Göbel U, Sitzmann FC. Impact Factor 2006: 1.055 - die klinische Pädiatrie im Spannungsfeld zwischen klinischer Forschung und Fortbildung. Klin Pädiatr 219: 249-251 